\title{
Taking Two-Photon Excitation (2PE) Further: 2PE Coupling to Far-field Optical Nanoscopy and Super Resolution Microscopy Towards Three-Dimensional (3D) Imaging of Thick Scattering Specimens
}

\author{
Alberto Diaspro ${ }^{1,2}$, Paolo Bianchini ${ }^{2}$, Francesca Cella Zanacchi ${ }^{2}$, Giuseppe Vicidomini ${ }^{2}$, Benjamin \\ Harke $^{2}$, Silvia Galiani ${ }^{1,2}$, Jenu Chacko ${ }^{1,2}$, Ivan Coto ${ }^{1,2}$, Zeno Lavagnino ${ }^{1,2}$, Marta d'Amora ${ }^{1}$ \\ 1. Istituto Italiano di Tecnologia, Department of Nanophysics, Genoa, Italy \\ 2. University of Genoa, LAMBS-Department of Physics, Genoa, Italy
}

Two-photon excitation (2PE) microscopy implementation in biological sciences and medicine [1] was immediately identified as a powerful fluorescence imaging method for studying living cell aggregates or tissues up to a significative depth, today close to one millimeter, not allowed by single photon approaches. Further benefits of two-photon excitation include: localized volume of excitation and emission, background rejection especially in 3D imaging, intrinsic optical sectioning, 3D localized photobleaching, photoswitching and uncaging, long term experiments [2]. These features have made possible experiments and innovations beyond the capability of traditional confocal microscopy. Unfortunately, one of the practical drawbacks in 2PE fluorescence microscopy is given by the worsening of spatial resolution due to red-shift of the excitation wavelength, only partially compensated by the improved signal-to-noise ratio. So far, image restoration [3] or aperture engineering [4] approaches have been used to alleviate such a condition.

In 1994, Hell and Wichmann introduced the stimulated emission depletion (STED) concept as a way to achieve optical far-field super resolution by circumventing the diffraction barrier [5]. This revolutionary approach has been also implemented under 2PE conditions [6]. Considering the advantages related to 3D-2PE fluorescence microscopy, a 2PE-STED architecture allowing to enhance spatial resolution performances will be discussed. Under such conditions, 1PE STED and 2PE STED are driven to the very same resolution performance having only the weakness given by the low efficiency of $2 \mathrm{PE}$ with respect to 1PE. Since one of the main goals, in medical and biological applications, is to address questions related to thick highly scattering specimens, we recently designed, implemented and demonstrated a SW (single wavelength)-2PE-STED approach [7].

Demonstration that a widely used red-emitting fluorophore, ATTO647N, can be two-photon excited at a wavelength allowing both $2 \mathrm{PE}$ and STED using the very same laser source, will be discussed. This fact allows to perform 2PE microscopy at four to five times STED-improved resolution, while exploiting the intrinsic advantages of nonlinear excitation.

In 2006 outstanding super resolution methods, based on precise localization of single molecules at the nanoscale, have been demonstrated following the pioneering PALM approach [8]. Such methods utilize the photophysical properties of fluorescent molecules by enabling the detection of sparse individual molecule photo-switched signatures. As a first step to 3D imaging in thick scattering cellular aggregates an individual molecule localization (IML) approach within a selective plane illumination microscopy (SPIM) set-up [9] has been implemented. IML-SPIM allowed to achieve 3D super resolution in tumoral mammalian cell spheroids [10]. 
In order to improve deep penetration imaging in highly scattering samples a 2P-SPIM approach will be addressed [11]. The effects induced by light-sample interactions and a comparison between the performances of $1 \mathrm{PE}$ and $2 \mathrm{PE}$ in scattering samples is quantitatively analyzed.

Following early demonstration of $2 \mathrm{P}$ photoactivation of photoactivatable fluorescent proteins [12], a 2P photoactivation-IML-SPIM will be critically discussed and demonstrated.

Such technological advances are expected to contribute to further growth of and access to multimodal bioimaging. An incredibly powerful battery of super resolution tools is available. Combining the features of different imaging methods and modalities enables to explore previously unseen aspects of biological systems at the nanoscale.

\section{References:}

[1] W. Denk, J. Strickler and W. Webb, Science 248 (1990) p.73.

[2] A. Diaspro et al. A., Q Rev Biophys 38 (2005) p. 97.

[3] P.P. Mondal et al., Applied Physics Letters 92 (2008) p.103902

[4] E. Ronzitti et al., Optics Express 17 (2009) p. 6867.

[5] S.W. Hell and J. Wichmann, Opt. Lett. 19 (1994) p. 780.

[6] G. Moneron and S.W. Hell, Opt Express 17 (2009) p.14567.

[7] P. Bianchini et al. PNAS 109 (2012) p. 6390.

[8] E.Betzig et al., Science 313 (2006) p.1642.

[9] J. Huisken, J. Swoger, F. D. Bene, J. Wittbrodt and E. H. K. Stelzer, Science 305 (2004) p. 1007.

[10] F. Cella Zanacchi et al., Nature Methods. 8 (2011) p.1047.

[11] Z.Lavagnino et al., Opt. Express (2013) in press.

[12] M. Schneider et al., Biophys J. 89 (2005) p.1346. 